\title{
Fundamentos pedagógicos para la creación y producción de recursos educativos abiertos (REA)*
}

\author{
Alexandra Gómez Marín** \\ Edilberto Restrepo Restrepo** \\ Ricardo Andrés Becerra Agudelo*** \\ Recibido: 2020-07-02 • Enviado a pares: 2020-07-20 \\ Aprobado por pares: 2020-08-05 • Aceptado: 2020-08-22 \\ https://doi.org/10.22395/angr.v19n38a3
}

\begin{abstract}
Resumen
En este artículo se presenta el componente pedagógico del modelo derivado de la investigación Modelo para la cocreación y producción de contenidos educativos abiertos mediante el uso de una plataforma virtual. Este modelo busca contribuir al fortalecimiento de la formación virtual en el departamento de Antioquia.

El objetivo de dicha investigación fue implementar un modelo para la cocreación y producción de contenidos educativos abiertos, llamados REA, mediante el uso de una plataforma virtual. Este modelo, que es el producto principal de esta investigación, está estructurado por tres componentes principales: pedagógico, de producción y tecnológico, los cuales se encuentran alineados a través de la autonomía, interacción, flexibilidad y mediación como principios transversales al modelo que lo orientan hacia la creación de contenidos educativos abiertos. El propósito fundamental de esta investigación es promover una educación en la cual se mejoren los procesos de enseñanza-aprendizaje a través de los REA haciendo uso de la tecnología y de los elementos necesarios para que los contenidos sean más eficientes y productivos. Al mismo tiempo, se busca implementar su distribución bajo licencias que permitan su reuso, adaptación y transformación sin restricciones de propiedad intelectual. El componente pedagógico, en el cual se hará énfasis en este artículo, surge del análisis de diferentes teorías, estrategias y metodologías de enseñanza-aprendizaje que se utilizan en la educación mediada con las tecnologías de la información y la comunicación (TIC). Este componente del modelo se fundamenta en el conectivismo como teoría de aprendizaje que fundamenta el proceso de creación de recursos educativos abiertos.

Palabras claves: modelo educacional; estrategias educativas; pedagogía; ciencias de la educación; tecnología de la comunicación; recursos educativos abiertos.
\end{abstract}

El artículo hace parte de la investigación Modelo para la cocreación y producción de contenidos educativos abiertos, mediante el uso de una plataforma virtual. Esta fue realizada como parte de la convocatoria para cofinanciar proyectos de I+D con el fin de contribuir al fortalecimiento de la formación virtual en el departamento de Antioquia con recursos del Sistema General de Regalías, Fondo de Ciencia Tecnología e Innovación de Colombia de Minciencias, la Universidad de Medellín y la empresa Asertiva Digital, Colombia.

* Licenciada en Pedagogía Infantil y magíster en Educación, Institución, Ciudad, País. Gestora pedagógica virtual de Educación Virtual y TIC, docente universitaria y miembro del grupo de investigación E-Virtual, Universidad de Medellín, Medellín, Colombia. Correo electrónico: algomez@udem.edu.co. Orcid: https://orcid.org/0000-0002-8145-9299

... Magíster en Educación Superior, Pontificia Universidad Javeriana, Bogotá, Colombia. Especialista en Gerencia de Proyectos, Universidad del Tolima, Ibagué, Colombia. Administrador de empresas, Institución Universitaria Ceipa. Medellín, Colombia. Gestor pedagógico de educación virtual y TIC e investigador del Grupo de Educación Virtual y TIC, Universidad de Medellín, Medellín, Colombia. Correo electrónico: oscar.restrepo@medellin.gov.co. Orcid: https://orcid.org/0000-0002-3456-6788

...* Magíster en Entornos Virtuales de Aprendizaje, Universidad de Panamá, Panamá. Ingeniero informático, Universidad Uniciencia, Bogotá, Colombia. Profesor de la Facultad de Comunicación e investigador del grupo de investigación E-Virtual, Universidad de Medellín, Medellín, Colombia. Correo electrónico: rbecerra@udem.edu.co. Orcid: https://orcid.org/0000-0002-5187-0482 


\title{
Educational Foundations for the Creation and Production of Open Access Educational Resources (REA)
}

\begin{abstract}
This article is the result of a research of the Model for the co-creation and production of open educational contents through a virtual platform (Modelo para la cocreación y producción de contenidos educativos abiertos mediante el uso de una plataforma virtual) This model aims towards making a contribution in the strengthening of digital education in the department of Antioquia. The goal of this research was implementing a model for the co-creation and production of open educational contents, REA in Spanish, through the use of a virtual platform. This model, that is the main product of this research, is restricted within three main components: educational, production and technologic, which are aligned through autonomy, interaction, flexibility and mediation as transversal principles that encompasses the creation of open educational contents. The fundamental purpose of this research is promoting an education in which the teaching-studying processes are improved through the REA by making use of technology and the elements necessary for making more productive and efficient contents. At the same time, the study aims to implement its distribution under licenses that allow its re-use, adaptation and transformation without intellectual property restrictions. The educational component, in which an emphasis is made in this article, emerges from the analysis of different theories, strategies and methodologies for teaching and learning that are used in mediated technology through information and communication (CIT). This component of the model is fundamented in connectivism as a learning theory that bases the creation processes of open educational resources.
\end{abstract}

Keywords: educational model; educational strategies; education; education sciences; communication technologies; open educational resources.

\section{Fundamentos pedagógicos para a criação e a produção de recursos educativos abertos (REA)}

\begin{abstract}
Resumo
Neste artigo, é apresentado o componente pedagógico do modelo derivado da pesquisa "Modelo para a cocriação e a produção de conteúdos educativos abertos mediante o uso de plataforma virtual". Esse modelo pretende contribuir para fortalecer a formação virtual em Antioquia, Colômbia. O objetivo desta pesquisa foi implementar um modelo para a cocriação e a produção de conteúdos educativos abertos, chamado de "REA", mediante o uso de uma plataforma virtual. Esse modelo, produto principal da pesquisa, está estruturado por três componentes principais: pedagógico, de produção e tecnológico, os quais se encontram alinhados por meio da autonomia, da interação, da flexibilidade e da mediação, como princípios transversais ao modelo que o orientam à criação de conteúdos educativos abertos. Assim, o objetivo fundamental desta pesquisa é promover uma educação na qual os processos de ensino-aprendizagem sejam melhorados por meio dos REA, com o uso da tecnologia e dos elementos necessários para que os conteúdos sejam mais eficientes e produtivos. Ao mesmo tempo, pretende-se implementar sua distribuição sob licenças que permitam seu reuso, adaptação e transformação sem restrições de propriedade intelectual. O componente pedagógico, no qual se faz ênfase neste artigo, surge da análise de diferentes teorias, estratégias e metodologias de ensino-aprendizagem que são utilizados na educação mediada com as tecnologias da informação e da comunicação. Esse componente do modelo é fundamentado no conectivismo como teoria de aprendizagem que subsidia o processo de criação de recursos educativos abertos.
\end{abstract}

Palavras-chave: modelo educacional; estratégias educativas; pedagogia; ciências da educação; tecnologia da comunicação; recursos educativos abertos. 


\section{Introducción}

En este artículo se presentará el componente pedagógico del modelo para la cocreación y producción de contenidos educativos abiertos, producto de la investigación Implementación de un modelo para la cocreación y producción de contenidos educativos abiertos, mediante el uso de una plataforma virtual. Aquí se da cuenta de uno de los objetivos específicos de la misma, el cual apunta a identificar metodologías de enseñanza y aprendizaje aplicadas para la cocreación y producción de contenidos educativos abiertos (CEA).

Asimismo, es el resultado de un proceso interdisciplinario de expertos en edu- cación, tecnología y comunicación que, desde sus saberes y experiencias, se encaminaron a la búsqueda y selección de metodologías y estrategias que, al integrar el quehacer pedagógico con las potencialidades tecnológicas, facilitaron significativamente el proceso de enseñanza-aprendizaje como aporte del componente pedagógico en la concepción e implementación del modelo planteado.

Los recursos educativos abiertos (REA) son elementos o materiales en formato digital compartidos en repositorios públicos para ser utilizados en procesos de enseñanza-aprendizaje por profesores, estudiantes e investigadores. La distribución de estos recursos se realiza mediante licencia Creative Commons para que puedan ser utilizados, adaptados y redistribuidos sin ninguna restricción (Unesco, 2002). Estos recursos son materiales educativos digitales construidos a partir de una intención pedagógica y formativa clara. El diseño y la manera en que se consolidan deberán responder enteramente a dicha concepción.

Como lo plantean Arango y Manrique (2020), los recursos educativos digitales abiertos son estos REA en formato digital que se distribuyen de forma libre para ser utilizados en procesos de enseñanza-aprendizaje. Estos recursos motivan, promueven y propician su uso, adaptación, actualización y personalización con fines formativos. Desde estos se pueden identificar rasgos e intenciones de la didáctica del profesor, tales como aspectos en favor de la comprensión y construcción de saberes en el estudiante.

La pretensión de esta investigación fue identificar un modelo que permitiera, a partir de una plataforma virtual, la producción de un REA que tengan en cuen- ta asuntos de orden pedagógico y tecnológico, y a la vez un procedimiento que facilitara y favoreciera la producción. Un modelo es "una reflexión anticipadora, que emerge de la capacidad de simbolización y representación de la tarea de enseñanza-aprendizaje, que los educadores hemos de realizar para justificar y entender la amplitud de la práctica educadora" (Mayorga y Madrid, 2010, p. 93). 
Ante el vacío identificado en relación con la claridad de un modelo que permitiera identificar un proceso para producir recursos educativos abiertos, esta investigación se propuso delimitar un modelo en cuyo centro se contempla al maestro y a otros actores del proceso educativo no solo como reproductores de contenidos diseñados por otros, sino como productores de su propio contenido, es decir, sujetos activos en el agenciamiento de los procesos de aprendizaje.

Este modelo se clasifica como un modelo didáctico tecnológico en tanto permite la combinación de estrategias que favorecen la construcción de conocimiento a través de metodologías activas. Según lo planteado por Bonwell y Eison (1991), al no encontrar una definición clara y puntual sobre lo que es el aprendizaje activo, procuraremos acercarnos desde los referentes encontrados sobre sus características metodológicas considerando que aprender activamente sugiere un compromiso con el objeto de estudio. De esta forma, el estudiante deberá, más que escuchar, leer, cuestionarse, escribir, discutir y aplicar conceptos, utilizar reglas y principios para encontrar solución a los problemas planteados. En este sentido, deberá exponerse a situaciones que le exijan procesos intelectuales de orden superior (analizar, evaluar, interpretar, inferir, crear).

En la elaboración de REA deben utilizarse metodologías activas que permitan desarrollar el aprendizaje activo. Si entendemos la metodología como la aplicación de un método y el método como el conjunto de operaciones ordenadas que conducen a la obtención de un objetivo, podríamos hablar en este ámbito, de una forma más apropiada, de estrategias promotoras de este aprendizaje, entendidas como el arte de coordinar actividades, recursos y acciones para alcanzar el aprendizaje activo de los estudiantes (Bonwell y Eison, 1991).

Al respecto de la promoción del aprendizaje activo, Freeman et al. (2014) plantean la importancia de aplicar métodos, como el aprendizaje cooperativo y colaborativo basado en problemas y proyectos, así como aprendizaje en equipos y el aula invertida, en tanto gran parte del aprendizaje activo se da dentro de las interacciones en grupo. De esta manera, el aprendizaje se constituye en un proceso más divertido y generador de lazos entre los miembros, lo que da lugar al razonamiento en su interior. El trabajo en grupo es concebido como la interdependencia positiva de los participantes hacia el logro de un consensuado objetivo común.

El aprendizaje activo también se ha convertido en uno de los métodos de instrucción más utilizados para el desarrollo de la gestión en organizaciones públicas y privadas (Boaden, 2006). El crecimiento del aprendizaje activo es atribuible a la noción de que los participantes aprenden mejor los nuevos comportamientos y las 
habilidades para resolver problemas a través de problemas del mundo real (Bowerman, 2003; Conger y Toegel, 2002).

La investigación del componente pedagógico del modelo se centró en la exploración de las posibilidades con las que cuenta la educación a partir de mediaciones relacionadas con la tecnología en espacios educativos no tradicionales (como las plataformas virtuales) entre los sujetos del acto educativo, lo cual precisa de la implementación de metodologías de enseñanza-aprendizaje propias para las demandas educativas del momento actual. Para ello, se vale de la producción de recursos educativos a partir de la idea de que una de las limitantes para un efectivo desarrollo de la educación a distancia bajo la metodología virtual es la elección de metodologías y estrategias pedagógicas que, salvando las debilidades heredadas de las formas tradicionales, permitan un mayor alcance en el proceso educativo.

Entre los referentes abordados, se destaca el conectivismo como la teoría del aprendizaje en la que se fundamenta el modelo. Para esta teoría, "el conocimiento queda constituido por la formación de conexiones entre nodos de información, ya sean estos contenidos aislados o redes enteras, y el aprendizaje consiste en la destreza para construir y atravesar esas redes" (Downes, 2007). El conectivismo parte de la idea de que el aprendiz debe tomar el control de su aprendizaje y operar en las redes sin maestros, sin instituciones reguladoras, sin presiones ligadas a la institucionalidad, sino solo a su propio deseo y motivación de formarse y aprender.

Según Siemens (2008), el conectivismo, como una teoría de aprendizaje para la actualidad digital, analiza las formas en que se aprende en una sociedad articulada en todo ámbito con las redes de conexión. Sustenta este aprendizaje en aspectos como la diversidad de opiniones, la conexión especializada de nodos o fuentes de información; la ubicación del saber en artefactos no necesariamente humanos, es decir, se enfoca más en la capacidad de conocer que en lo conocido; la relevancia de identificar las conexiones en el aprendizaje continuo; la habilidad para conectar áreas, ideas y conceptos esenciales; la toma de decisiones como proceso de aprendizaje; y en la determinación adecuada del que aprende, en su significado entrante y en la capacidad de contrastarlo en una realidad cambiante.

Bajo este modelo, las propuestas pedagógicas que se generan parten del conectivismo en tanto permite motivar y entrenar de manera gradual al estudiante para que tome el control de su propio aprendizaje, de tal forma que el profesor se constituya en guía del proceso y el estudiante vaya conformando una red personal y colaborando en los procesos de creación conjunta de saberes. Con ello, se logra promover el pensamiento crítico y la creatividad para el uso de herramientas de publicación de contenidos que favorezcan la reflexión sobre la propia formación. 
El proceso de aprendizaje se entiende, en su matiz conectivo e interactivo, como aquel donde se desarrollan habilidades en el uso de las tecnologías y escenarios de interacción, colaboración, participación y conexión de nodos hasta obtener la autosuficiencia y la autonomía en la creación de redes de conocimiento para un aprendizaje autorregulado, esto es, que se involucre y motive en un aprendizaje permanente y móvil para que utilice los medios fuera de las aulas tradicionales.

El principal resultado de la investigación fue el modelo de plataforma virtual que permite la creación de los recursos educativos abiertos bajo perspectivas claras de funcionalidad, variables críticas, generación de actividades de e-learning y una estructura nodal que, al tiempo, servirá como repositorio para los RED. De esta forma, quienes la utilicen pueden construir sus propios recursos o puedan buscar, entre los ya evaluados, uno que cumpla con las necesidades y las actividades necesarias para su objetivo académico.

En lo relacionado con el componente pedagógico de dicho modelo se encontraron diferentes metodologías y estrategias educativas implementadas en el campo de la producción de REA. En este componente, después de un análisis riguroso, se delimitan aquellas metodologías y estrategias que se privilegian en la concepción y producción de los recursos elaborados en dicha plataforma. Valga destacar que cada modelo o estructura para la producción de REA fundamenta su proceder en los principios pedagógicos que de acuerdo con la tradición epistemológica que consideran más pertinentes.

Otro asunto que también se encontró como fundamental se relaciona con las capacidades con que cuenta cada estudiante para acogerse a las dinámicas que implica la educación mediada con las TIC. Por lo tanto, es necesario contar con la formación que no solo requieren quienes están en el rol de productores de contenido, sino también con las competencias comunicacionales y cognitivas en la población estudiantil.

La incorporación al proceso educativo de unas estrategias y metodologías pedagógicas que favorezcan el aprendizaje y su mediación a través de entornos virtuales es fundamental para darle lugar a un proceso formativo sólido. A continuación, se dará cuenta de las estrategias y metodologías que se hallaron en la investigación, las cuales se privilegian para consolidar procesos educativos de mayor calidad. Igualmente, se presentará en qué consiste el componente pedagógico que emergió como resultado al consolidar el modelo para la cocreación y producción de contenidos educativos abiertos. 


\section{Metodología}

En el marco más amplio del proyecto de investigación sobre la implementación de un modelo para la cocreación y producción de contenidos educativos abiertos mediante el uso de una plataforma virtual se desprenden objetivos orienta- dos a la identificación de metodologías y estrategias de enseñanza y aprendizaje. Sin embargo, la caracterización de las didácticas y técnicas utilizadas en la producción de contenidos educativos; el establecimiento del software y hardware; la definición de lineamientos metodológicos; las herramientas digitales para la cocreación y producción de recursos educativos abiertos; el desarrollo de una plataforma virtual; y este documento, como publicación de un avance de este proyecto, es dar a conocer los hallazgos del primer objetivo: "identificar metodologías y estrategias de enseñanza-aprendizaje propicias para la cocreación y producción de REA (recursos educativos abiertos)". Este objetivo se refiere procesos metodológicos necesarios para el logro de este objetivo específico, lo que constituye el componente pedagógico que, sumado al tecnológico y al de producción, sustentan el modelo que se va a implementar en la investigación.

Partiendo de lo anterior, el componente pedagógico se orienta a la búsqueda de estrategias y metodologías de enseñanza-aprendizaje que consideren los saberes previos, propicien el uso tecnológico, la construcción individual y colectiva de conocimiento, las interacciones dinámicas con los contenidos y con otros, y potencien las características de usabilidad y naturaleza misma de los recursos educativos abiertos como elementos mediadores para el autoaprendizaje a partir de un análisis que identifique el todo y cada una de sus partes. Es así como las características de estas necesidades de búsqueda llevan a plantear una metodología basada en los principios de la hermenéutica y la perspectiva cualitativa.

Se entiende la hermenéutica como el arte de interpretar textos sagrados, filosóficos o literarios en busca del verdadero significado de las palabras escritas o verbales, donde toda labor de conceptualización persigue el consenso, el acuerdo posible e, incluso, debe basarse en un consenso si se ha de lograr que las personas se entiendan entre sí (Gadamer, 1992, p.116). En este sentido, es importante comprender e interpretar el discurso desde la exposición del autor para proponer una interpretación aún mejor. También es importante entender la perspectiva cualitativa de la investigación según la cual, como lo propone Galeano (2004) "el conocimiento es un producto social y su proceso de producción colectivo está atravesado por los valores, percepciones y significados de los sujetos que lo construyen" (p. 18). Es así como se desarrolla el componente pedagógico referido a las metodologías, didácticas y estrategias de enseñanza aprendizaje para la creación de los recursos educativos abiertos. 


\section{Enfoque metodológico bajo el círculo hermenéutico}

Desde lo anterior y bajo los elementos en los que se enmarca el proceso hermenéutico, se desarrollaron fases que referían los prejuicios, el análisis, la comparación, la reflexión, la comprensión, la interpretación y la síntesis en el procesamiento de los datos recolectados. En principio se identificaron las necesidades y las fuentes documentales para luego acercarse a ellas en una búsqueda intencionada de información aportante con el fin de conceptualizar, decantar y acercarse a los elementos esperados. En este sentido, afirma González (2006):

Traducción, prejuicios, análisis, comprensión, interpretación y síntesis, he ahí los conceptos básicos de una propuesta para desarrollar la hermenéutica como un proceso de lectura de lo real; además, se convierte simultáneamente en una posibilidad para la formación, para que cada quien construya su imagen y la exprese a través de los lenguajes. (p. 42)

De esta forma, el enfoque hermenéutico se lleva a cabo mediante: i) los prejuicios, como saberes previos de los investigadores y su experiencia de investigación que permitieron su transformación en nuevo conocimiento; ii) el análisis, al identificar el todo y cada una de sus partes como integralidad, donde las apreciaciones o alteraciones causadas de manera particular en el proceso de exploración podrían sugerir o llevar a cambios en la generalidad pretendida; iii) la comparación, que se da al confrontar los prejuicios de los investigadores y lo encontrado en los textos y referentes como fuentes del rastreo documental. Esto se da en una acción propuesta por González (2006) frente a este método hermenéutico, quien concibe:

Texto es, en sentido amplio, toda acción susceptible de traducción y, en sentido restringido, toda fijación que genera una estructura de sentido [...]. El texto es un todo, pero puede relacionarse con otros textos y comprenderse intertextualmente. Al texto en sí, lo acompaña el conjunto de los otros textos, de los otros discursos, de las otras acciones que también están inscritas en él. (p. 43)

Los demás elementos que conforman el enfoque hermenéutico son: iv) la reflexión que, desde los textos abordados y según las fichas construidas como reseña de cada uno, se extrajo del investigador que condujo a indagar sobre las teorías, estrategias, metodologías de enseñanza y aprendizaje aplicadas en la creación individual o colectiva de recursos educativos abiertos, así como las características didácticas y las técnicas utilizadas para este propósito; v) la comprensión, como proceso esencial de la investigación cualitativa frente a la presentación de los resultados, donde se conocen los nuevos textos y se convalida la relevancia de los elementos iniciales como prejuicios; vil la interpretación o proyección de lo comprendido, en una relación de los textos con el contexto de la investigación La creación y producción de recursos 
educativos abiertos, publicados en plataformas e-learning, como aquellas conexiones que logra el investigador en la lectura del texto y la atribución de significados de los elementos técnicos y pedagógicos que fluyen durante el proceso de comprensión; vii) la síntesis, en un cierre del círculo hermenéutico, se plantea con la creación de un nuevo texto resultante de todo este proceso descrito, en el que puede ubicarse un producto que da cuenta de las metodologías, estrategias y didácticas de enseñanza y aprendizaje aplicadas para la creación individual y conjunta de recursos educativos abiertos (REA), como resultado del objetivo planteado para su identificación desde el planteamiento inicial del proyecto de investigación.

\section{Desarrollo metodológico}

El ejercicio desarrollado a la luz de los elementos del enfoque hermenéutico planteado y hacia la identificación de estrategias y metodologías de enseñanza y aprendizaje propicias para la creación de recursos educativos abiertos, condujo a la exploración bibliográfica de aquella literatura que, en sus diferentes ámbitos y propuestas, permitiera consolidar los resultados esperados. Fue así como, partiendo de los conceptos previos desde la experiencia y formación de los investigadores, se inicia este proceso al determinar las fuentes de búsqueda según su nivel de cientificidad, línea de profundización temática, posibilidades de acceso, idioma y lista de reconocimiento a nivel nacional e internacional. Así, las fuentes de búsqueda fueron libros y artículos publicados en bases de datos con información sobre teorías de enseñanza-aprendizaje, estrategias y metodologías didácticas, por lo que las consultas exploratorias fueron realizadas en fuentes ubicadas en Google Scholar, Redalyc, Jstor, Springer, Elsevier, Link, Taylor \& Francis, Eric y Scopus.

\section{Criterios definidos para la depuración de las fuentes y la información}

Para iniciar la búsqueda y exploración de las fuentes definidas se determinan, mediante la lluvia de ideas y desde la claridad del contexto, el objetivo de la investigación y el objetivo específico sobre las estrategias y metodologías para la enseñanzaaprendizaje. A partir de un conjunto de criterios representados por cadenas de texto, fechas, idiomas y áreas del saber específico, se avanza con una depuración basada en la pertinencia y cercanía con los propósitos de la búsqueda, en lo que se inicia un acercamiento a las fuentes señaladas bajo estos criterios.

En lo que podría considerarse el universo de documentos referidos a las temáticas o áreas del saber en las metodologías y estrategias de enseñanza-aprendizaje para obtener documentos con un mayor nivel de aporte a nuestro objetivo, se consignan para su aplicación en un formato planilla los siguientes criterios: 
Cadenas de búsqueda en las bases de datos: "cocreación + metodologías de enseñanza o metodologías de aprendizaje", "cocreación + estrategias de enseñanza o metodologías de aprendizaje", "estrategias o metodologías de enseñanza", "estrategias o metodologías de aprendizaje", "cocreation + teaching methodologies o learning methodologies", "contenidos educativos abiertos + diseño o creación", "open educational resources + design".

\section{Parámetros de inclusión-exclusión}

Como filtro de los resultados obtenidos mediante cada cadena de búsqueda aplicada por la pertinencia y actualidad de los contenidos, se tomarán en cuenta solo aquellos publicados a partir del año 2013. Por las posibilidades de comprensión e interpretación de los investigadores y la alta posibilidad científica de textos en estos idiomas, se acogen solo textos en inglés y español. Además, dado que el estudio se realiza para la implementación de un modelo para la educación superior, se dará alta prioridad a las publicaciones de investigaciones realizadas para este nivel educativo. Pero también se toman, en un grado muy bajo, aspectos más genéricos de investigaciones en otros niveles académicos.

\section{Universo y muestra de los referentes abordados}

Como universo o, de alguna manera, la población de referentes — potenciales objetos de análisis para la obtención de la información significativa para la investigación-, se parte de las bases de datos elegidas por el perfil y tipificación en sus líneas de conocimiento que tuviesen mayor tendencia a la temática que orienta el proyecto. De allí se ubican aquellos documentos que, al respecto de los criterios establecidos para la depuración y refinamiento de los mismos, tuvieran mayor ocurrencia, inicialmente en su título o resumen. De esta manera, se realiza un primer filtro en este universo de documentos para obtener una visión general de la literatura científica sobre la temática deseada. Se obtiene, entonces, lo que podríamos denominar población efectiva: quinientos diez documentos científicos con alguna referencia a las metodologías y estrategias para la enseñanza-aprendizaje en los procesos de cocreación de recursos educativos abiertos.

La muestra efectiva de la población anterior de documentos se obtiene mediante un acercamiento de exploración a las temáticas que dieran cuenta, desde sus títulos, de los contenidos de a cada documento, de tal forma que desde su introducción o temas contenidos pudiese evidenciar un aporte significativo a los propósitos de la investigación. De ello, se obtuvo una muestra de doscientos quince referentes en formas de publicación variada como artículos e informes de investigación, artículos 
de opinión y capítulos de libro con información relevante y aportante frente a las estrategias y metodologías propias para la enseñanza aprendizaje orientadas al trabajo autónomo, aprendizajes significativos y la colaboración.

\section{Instrumentos aplicados en la obtención de la información}

Los instrumentos para el registro de la información encontrada bajo los criterios definidos fueron constituidos con campos que permitieran mantener presente el contexto y el objetivo sobre el cual se enfoca la información a obtener, lo que dio lugar a diversos formatos que permitieron almacenar y depurar la información.

\section{Planilla de registro de revisión bibliográfica}

Como instrumento para la recolección de esta información, se dispone en un formato planilla el objetivo general de la investigación y el objetivo específico que nos convoca en esta exploración, las cadenas de búsqueda en primera columna y, a nivel de cabecera, cada una de las bases de datos dispuestas como fuentes de indagación. Esto se hace con el fin de determinar, en primera instancia, el número de documentos que como población cumplan con los criterios establecidos. De esta manera, se obtiene como registro de este instrumento una visión general de la existencia de literatura científica en la que se dé cuenta de las metodologías y estrategias de enseñanza-aprendizaje, asociada o propicia para la cocreación de recursos educativos abiertos.

darse dio por finalizado el proceso de exploración de literatura bajo el cumplimiento de los criterios planteados en las fuentes dispuestas y al análisis de pertinencia temática que cada investigador pudiera encontrar en el título de cada documento, lo que determinó en última instancia su registro en el instrumento de recolección.

\section{Ficha de revisión documental}

A partir de cada uno de los documentos obtenidos en la última versión de la planilla de registro de revisión bibliográfica, se analizó su contenido en busca de elementos que permitieran determinar aspectos puntuales frente a las teorías de aprendizaje, el rol del estudiante y el profesor, la disposición de los contenidos, la formas de interacción, los recursos utilizados, los propósitos e intenciones didácticas, las tipologías de actividad, bien individual o colectiva, y sus posibilidades de aplicación en una entorno de aprendizaje como el que brinda una plataforma virtual. Desde estos elementos, se buscó determinar estrategias o metodologías propicias para la enseñanza-aprendizaje mediante el uso de recursos educativos abiertos. 
En función de lo anterior, se registra en la ficha de cada documento: i) la información general, el autor(es), título, subtítulo, editor, edición, ubicación del texto url/doi, referencia bibliográfica en formato APA, tipo de documento; ii) resumen, una breve descripción del contenido del documento que permite identificar los elementos esencialmente aportantes, iii) información tomada de la fuente, texto que puede citarse directamente para la investigación, iv) notas del investigador, fecha y nombre del investigador que elaboró la ficha y fecha de análisis del documento.

Las notas del investigador son de gran relevancia en tanto se consigna allí el conocimiento nuevo o relacionado con sus prejuicios. Esto podría orientarse a un producto no solo pensado en la enseñanza, sino en la posibilidad de generar un aprendizaje basado en unos principios básicos y recomendaciones de autoaprendizaje.

La teoría que se obtiene de la literatura es una plantilla para comparar, de modo que el investigador pueda reconocer qué es nuevo y emocionante de manera instantánea cuando ve algo conocido. El propósito es convertirse en un investigador astuto e inteligente, no dirigido, para conseguir la máxima consciencia y reconocer los caminos. Así, cuando se entra al medio, el investigador se basa en la capacidad de ver la experiencia desde la perspectiva del participante, lo cual es la clave para una buena investigación cualitativa (Bottorff y Morse, 2003, p. 33). Una vez se han seleccionado los estudios primarios, se inicia la extracción de información relevante. Para ello, se diseña el siguiente instrumento como partida para el tratamiento de la información finalmente consolidada:

\section{Tratamiento de la información}

Sobre los documentos referidos en las fichas y como resultado del acercamiento inicial a los contenidos, se realiza un análisis que da lugar a la generación de categorías o elementos dominantes desde los que puedan plantearse aspectos de identificación, tipificación y argumentación frente a las estrategias y metodologías propias para la enseñanza-aprendizaje mediante el uso de recursos educativos abiertos en una plataforma virtual. Estas categorías se disponen en un instrumento de caracterización para la construcción y escritura de los resultados.

\section{Ficha de caracterización:}

Ya seleccionados los estudios primarios, se avanza con la extracción de información relevante al definir en la ficha las categorías que constituyan, desde lo general, los ejes contenedores para la escritura de los nuevos textos que resultan del objetivo de identificar estrategias y metodologías de enseñanza-aprendizaje. 


\section{Análisis de la Información recopilada}

Si bien desde el inicio del proceso de búsqueda de la información y durante los procesos de depuración para la obtención de la muestra de documentos se daban vestigios de análisis, se denota con mayor fuerza de análisis intencionado y efectivo hacia la obtención de los resultados desde el proceso que se inicia con el registro de la información en las fichas bibliográficas. Esto se debe a que es allí donde realmente se abordan los documentos de la muestra con una lectura más allá de la exploración, en una indagación para identificar teorías, planteamientos, elementos y argumentos desde los cuales puedan determinarse estrategias o metodologías propicias para la enseñanza-aprendizaje mediante el uso de recursos educativos abiertos.

Para adentrarse en las fuentes u objetos de revisión, y bajo las pistas o aspectos de identificación registrados en las fichas bibliográficas con alguna certeza de depuración efectiva, se determina que, para la consolidación de escritos que dieran cuenta de las metodologías y estrategias buscadas, se priorizan los que fundamentan, desde lo pedagógico, ideas, planteamientos, citas, argumentos y aportes en los que sus autores o sus referidos exponen roles del profesor y el estudiante, formas de presentación de los contenidos, didácticas, métodos, utilización de recursos, dinámicas de interacción y formas e instrumentos de evaluación aportantes a las estrategias y metodologías que favorecen el aprendizaje autónomo, el trabajo colaborativo y la interacción para la construcción conjunta de saberes.

Además, con la base de conocimientos previos del investigador frente a las teorías que enmarcan estas estrategias y metodologías desde la pedagogía y la didáctica, se avanza en la construcción de textos que recogieran, de forma estructurada, intencionada, coherente y enlazada, insumos que van caracterizando y particularizando estas formas de enseñanza-aprendizaje.

En una fase de análisis más orientada a la presentación de los resultados finales, se conjuga con mayor fuerza el saber pedagógico, metodológico y estratégico que pueda tener el investigador frente al proceso de enseñanza aprendizaje. Así, en una mirada crítica de los textos construidos en el proceso de tratamiento y caracterización de la información, se inicia una revisión y complemento de los mismos en busca de obtener contenidos más argumentados y amplios en sus concepciones. Para ello, la búsqueda se orienta hacia aquellas posturas o planteamientos que puedan brindar concepciones diferentes o variaciones en las metodologías encontradas, esto es, confrontaciones, críticas o potencialidades que los autores de esta muestra documental seleccionada pudiesen exponer al respecto. 
En la conjugación de los anteriores niveles de análisis, se logran obtener documentos en los que se plantean opiniones y posturas del investigador. Estas posturas, enlazadas con la intencionalidad de la investigación y la información obtenida, permiten obtener los resultados buscados para integrar las estrategias y metodologías de enseñanza aprendizaje que favorezcan el aprendizaje autónomo, el trabajo colaborativo, la construcción conjunta de saberes, el aprendizaje significativo y la cocreación de recursos educativos abiertos, con la mediación de una plataforma virtual de Aprendizaje.

\section{Resultados}

El modelo para la creación y producción de REA mediante una plataforma virtual, producto de esta investigación, cuenta con tres componentes: el tecnológico, el de producción (de los cuales se dio cuenta al principio de este artículo) y el pedagógico, en cuya articulación y sincronía se logra la realización de dichos recursos. Pero, al proponer un modelo que tendrá aplicación en el área educativa, es fundamental configurar y destacar su componente pedagógico. De acuerdo con este componente, tendrá lugar y orientación el tipo de recursos educativos que se van a producir bajo su lineamiento. A continuación, se presentará en qué consiste dicho componente. Mientras tanto, tras la revisión amplia de la literatura encontrada alrededor del tema central del modelo, se logra figurar un componente que cuenta con la educabili- dad del sujeto cuidadoso de atender a través de él las particularidades e implicaciones de la educación en ambientes mediados por las TIC.

El componente pedagógico del modelo se fundamentó en diferentes estrategias pedagógicas que ayudaron a desarrollar y fortalecer elementos que forman este componente, en tanto facilitan a los estudiantes adquirir el conocimiento necesario para la solución de situaciones problemáticas que se les presentarán a lo largo de su formación y luego podrán ser aplicadas en su vida laboral. Del mismo modo, la fundamentación de este componente permitirá a los profesores aprender nuevas técnicas para el desarrollo de los contenidos temáticos de sus asignaturas. Una de esas estrategias es el análisis de casos, reconocida por ser una de las estrategias más utilizadas en el aula de clase. Según Labrador y Andreau (2008), el análisis de casos:

Es un método flexible que permite la utilización de otras técnicas y proporciona al profesor completa libertad a la hora de introducirlo en la programación de su asignatura, así como secuenciarlo según las características y necesidades de sus alumnos. El éxito del método, fundamentalmente, depende de la competencia del profesor en su utilización y en una selección adecuada a los objetivos establecidos. (p. 37) 
El planteamiento de casos de estudio debe ser claro y conciso. Además, debe estar acompañado de documentos e información de soporte para que los estudiantes planteen la solución al conflicto propuesto y detallen los posibles caminos para la solución final que los lleve a obtener sus propias conclusiones. Con esta estrategia, los estudiantes trabajan en equipo y de forma colaborativa. Así, generan la posibilidad de expresar sus puntos de vista y de analizar las diferentes situaciones que se presentan en el desarrollo del caso.

Otra de ellas es el aprendizaje basado en problemas, también conocido como ABP. Esta estrategia permite a los estudiantes, a través de una problemática planteada por el profesor, adquirir y apropiar nuevos conocimientos que busquen la solución al problema en cuestión. Son muchas las definiciones que distintos autores han elaborado sobre el ABP, como la que ofrece McGrath (2002), donde lo define como un "método de aprendizaje en grupo que usa problemas reales como estímulo para desarrollar habilidades de solución de problemas y adquirir conocimientos específicos" (p. 10). Por otro lado, Gil-Galván (2018) afirma:

En el ABP la situación problematizada se propone como un reto que estimula la motivación de los estudiantes, apreciamos que con su puesta en práctica se persigue fomentar un tipo de aprendizaje que adelante el umbral del tradicional o convencional, limitado, en la mayoría de los casos, a la reproducción del conocimiento y, como mucho, a la interpretación relacionada con datos e informaciones de índole relevante. (p. 76)

El uso de esta estrategia permite a los estudiantes que, frente a una problemática trazada, discutan, planteen y reflexionen sobre diferentes formas de solucionar dicha problemática y generen en el salón de clase la posibilidad de organizar debates constructivos sobre las situaciones que se presentan en la vida personal y profesional de los participantes.

En la era digital que actualmente estamos viviendo es habitual que los estudiantes, especialmente los más jóvenes, interactúen en internet a través de diferentes espacios de comunicación para construir colectivamente el conocimiento, tal y como se realiza en redes sociales y comunidades virtuales. Según Siemens (2006), el aprendizaje en red es continuo y el conocimiento se crea y configura gracias a la actividad combinada que se da entre las personas. Conocer en la actualidad significa estar conectado, en constante dinamismo. El conocimiento está en la red y es abundante. Precisamente, esto es lo que hace necesario un buen número de tareas que debe cumplir todo docente: detectar lo importante, guiar los procesos de búsqueda, analizar la información encontrada, seleccionar, interpretar los datos, sintetizar el contenido y difundirlo. 
En el aula de clase es evidente el uso de dispositivos móviles. Por ello, los profesores están llamados a generar nuevas estrategias pedagógicas que permitan a los estudiantes participar y adquirir nuevas habilidades a través del aprendizaje en red, por ejemplo, mediante el uso de entornos virtuales donde los profesores publican contenidos educativos dinámicos que faciliten al estudiante la posibilidad de acceder al conocimiento desde cualquier dispositivo con conexión a internet. A través de los diferentes recursos de comunicación que ofrecen estas plataformas, se genera comunidad en línea para que todos participen en la construcción del conocimiento.

El aprendizaje basado en equipos es otra de las estrategias para destacar. Este promueve en los estudiantes la toma de decisiones y, a su vez, la adquisición de conocimiento sobre los conceptos propuestos por el profesor gracias a la cantidad de información que pueden manipular para dar solución a una problemática. Autores como Michaelsen, Davidson y Major (2014, pp. 57-84) lo definen como un aprendizaje que se genera a través del trabajo cohesionado de grupos heterogéneos de estudiantes que van logrando mayores grados de autonomía y de responsabilidad en la medida en que la estrategia se replica durante el curso académico. De acuerdo con lo que afirman Reyes-Torres, Saavedra y Aguayo-Vergara (2020), "el aprendizaje basado en equipos es utilizado ampliamente por docentes universitarios quienes han observado un mejor desempeño de sus estudiantes en la comprensión de los contenidos del curso, así como en el desarrollo del pensamiento crítico" (p. 4). Pero para su correcta implementación es necesario que el profesor propicie instancias de retroalimentación permanente y diseñe tareas que consideren las siguientes características: desafiantes para el desarrollo de los pensamientos complejos, precisos y breves que movilicen distintos conocimientos e impliquen toma de decisiones.

La aplicación del aprendizaje basado en equipos tiene éxito gracias a la formación e interacción en grupos reducidos, entre cuatro o siete integrantes. Es recomendable que los participantes tengan diferencias conceptuales y potenciales entre ellos para obtener diversas apreciaciones y perspectivas frente al problema. Las diferencias personales pueden determinarse por experiencia, conocimiento, practicidad, demografía, entre otras. Esto permite a los estudiantes adquirir nuevos saberes a través de la organización y gestión de los equipos de trabajo, lo que facilita el trabajo colaborativo para el desarrollo de la problemática propuesta y permite la consulta de información y la discusión entre los miembros del equipo para fundamentar la solución final de la actividad. 
Según la autora Gil-Galván (2018), en las comunidades de aprendizaje

se pueden discernir dos usos principales; el primero se centra en el elemento humano de las comunidades, y los beneficios que se acumulan a partir de las sinergias de las personas en lugares comunes o con intereses co- munes a medida que trabajan para compartir entendimientos, habilidades y conocimientos con fines compartidos; el segundo se centra en las estructuras curriculares (es decir, una estructura inanimada) como medio para desarrollar un aprendizaje más profundo de contenido curricular predeterminado (implícito). (pp. 73-93)

Esto implica que las comunidades de aprendizaje se crean, generalmente en línea, para que sus miembros se reúnan utilizando diferentes tipos de canales de comunicación asincrónica como foros, correos electrónicos o publicaciones con el fin de compartir un interés común o ayudar a otros a solucionar inquietudes o dudas sobre el tema en cuestión. Además, permiten solucionar inquietudes a los miembros de la comunidad que plantean inquietudes sobre una temática específica. En este sentido, Gairín (2006) afirma:

Las comunidades de aprendizaje son comunidades personales, al tratar con personas con intereses individuales, afinidades y valores, que utilizan la red en función de una temática específica. Serán más exitosas, por tanto, cuanto más estén ligadas a tareas, a hacer o a perseguir intereses comunes. (p. 51)

Generalmente, estas comunidades suelen ser abiertas para que cualquier persona pueda leer las publicaciones de los miembros y así encontrar en ellas la solución que necesite. Pero si el visitante desea participar en la comunidad para plantear una nueva problemática o queriendo dar respuesta a alguna publicación, es necesario que haga una inscripción previa para poderlo realizar. Los administradores de estas comunidades deben plantear objetivos claros sobre el manejo de la comunidad, lo cual permitirá una correcta interacción de sus miembros para poner a los participantes en una constante formación e intercambio creativo.

El aula invertida es otra estrategia para utilizar en el aula que busca que los estudiantes tengan autonomía en el aprendizaje. En esta estrategia el profesor propone contenidos teóricos que el estudiante revisa y estudia antes de ingresar al aula de clase, lo que permite que los estudiantes exploren e investiguen sobre los contenidos que se dictarán en su siguiente clase antes de que sea impartida por el profesor.

Para Schneider et al. (2013, pp. 68-81), el aula invertida es una metodología que cambia el orden de una clase tradicional. La presentación del contenido se realiza antes de la clase presencial por medio de videos breves, audios, lecturas, entre 
otros insumos. La clase presencial está centrada en la realización de actividades donde se utiliza el contenido abordado previamente por los estudiantes. A partir del reconocimiento de la importancia del dominio del contenido, la comprensión ampliada se alcanza con la mediación docente al momento de resolver la tarea. En este sentido, Cornide-Reyes y Villarroel (2019) reflexiona:

El aula invertida es un modelo didáctico-pedagógico que incluye actividades de aprendizaje significativo, personalizado y colaborativo que se realiza en el aula, e instrucción individual directa realizada en computador fuera del aula. Una característica importante de este modelo es el cambio en los roles profesor-estudiante con respecto al modelo tradicional. Es decir, el profesor asume un rol de guía, facilitador y colaborador durante el proceso de aprendizaje de los estudiantes y deja de ser la fuente única de conocimiento. Los estudiantes por su parte asumen un rol activo y protagónico en el proceso, ya que no solo son responsables de la adquisición y asimilación de los contenidos de manera previa a la clase, sino que deben participar en las diversas actividades prácticas realizadas en el aula en colaboración con sus pares. (p. 4)

En esta estrategia, el estudiante se convierte en el actor protagónico del proceso de enseñanza y el profesor en el guía que lo asesora. El material previo puede ser publicado en una plataforma virtual o comunidad en red o compartido a través de medios de almacenamiento en línea o correo electrónico con el fin de que los estudiantes puedan acceder fácilmente desde su casa o un lugar diferente a la institución educativa. El profesor genera espacios de debate como foros o encuestas, donde los estudiantes, después de leer el material, generan cuestionamientos o inquietudes que luego serán discutidos en el aula de clase.

La educomunicación es la estrategia que combina la educación y la comunicación, y surge del alto posicionamiento de la tecnología en todos los procesos educativos actuales. Con esta estrategia, el modo de aprender se enfoca en el análisis de teorías a través de una mirada crítica, integradora y comprometida con los intereses de cada persona.

Aplicar esta estrategia en el aula clase conlleva a que, a partir de los objetivos de la asignatura, los estudiantes tomen las riendas y decidan qué ruta de aprendizaje seguir. Con la información, datos y materiales recolectadas se discuten o proponen nuevas estrategias experienciales para ser evaluadas por sus profesores. La participación y desarrollo de la actividad obliga a los estudiantes a tener una actitud colaborativa en el trabajo en equipo. En este sentido, usan dinámicas de aprendizaje a través de la cocreación para generar las ideas que ayuden a conceptualizar las teorías y a generar nuevos conocimientos que fomenten el diálogo entre los participantes. 
El aprendizaje-servicio, conocido con las siglas APS, es una forma de enseñar realizando servicio a la comunidad. Con este, se busca afectar una necesidad social donde los estudiantes aprendan en acción y apliquen conocimientos, habilidades y competencias para el bien común. De acuerdo con Paredes (2019) "las prácticas de ApS están caracterizadas por la planificación intencional de los contenidos formativos y las actividades que configuran el servicio que se va a implementar en la comunidad, con el propósito de mejorar alguna dimensión de esta" (p. 27).

Según Martínez et al. (2013, pp. 195-216), el aprendizaje-servicio es una metodología que se basa en la integración entre el aprendizaje basado en la experiencia y el servicio que contribuye a dar soluciones reales frente a una problemática comunitaria. Utilizar la estrategia de aprendizaje y servicio permite que los estudiantes trabajen a favor de la comunidad. Asimismo, estos reciben un doble aprendizaje debido a que se adquieren los conocimientos de acuerdo con los objetivos de la asignatura y valores éticos y cívicos que se observan en todo el desarrollo de la actividad. Este beneficia tanto a los estudiantes como a los ciudadanos. Además, esta estrategia permite que los estudiantes se acerquen al mundo real y a que resalten su capacidad creativa y emprendedora en la solución de problemáticas sociales.

En el planteamiento del modelo y en el propósito fundamental de la investigación que da origen a este artículo es importante aclarar que se hallaron dos componentes más que se requieren tener en cuenta al producir REA. El primer componen- te es el tecnológico, el cual da cuenta de los requerimientos a nivel de software y hardware que permiten su elaboración. En este caso se cuenta con el apoyo de una plataforma virtual que orienta esta elaboración, de manera que quien desea realizarlo no sienta que parte de cero, sino que está respaldado por un proceso que ha sido definido y validado previamente por los investigadores. El segundo componente es el de producción. Este se refiere a la cadena de producción que es preciso delimitar para llevar a buen término la realización de un REA, es decir, el talento humano que se requiere, pues se trata de un equipo interdisciplinario que interactúa con el contenido plano y define las mediaciones pedagógicas y didácticas necesarias para que dicho contenido se constituya en un REA capaz de responder a la intención pedagógica que tiene. Lo anterior se condensa de mejor manera en la figura 1.

Además de sus tres componentes, el modelo está concebido a partir de cuatro principios, es decir, cuatro puntos de partida para la creación y producción de los recursos educativos abiertos indispensables a la hora de emprender dicha tarea. Estos principios son: interacción, mediación, autonomía y flexibilidad. 


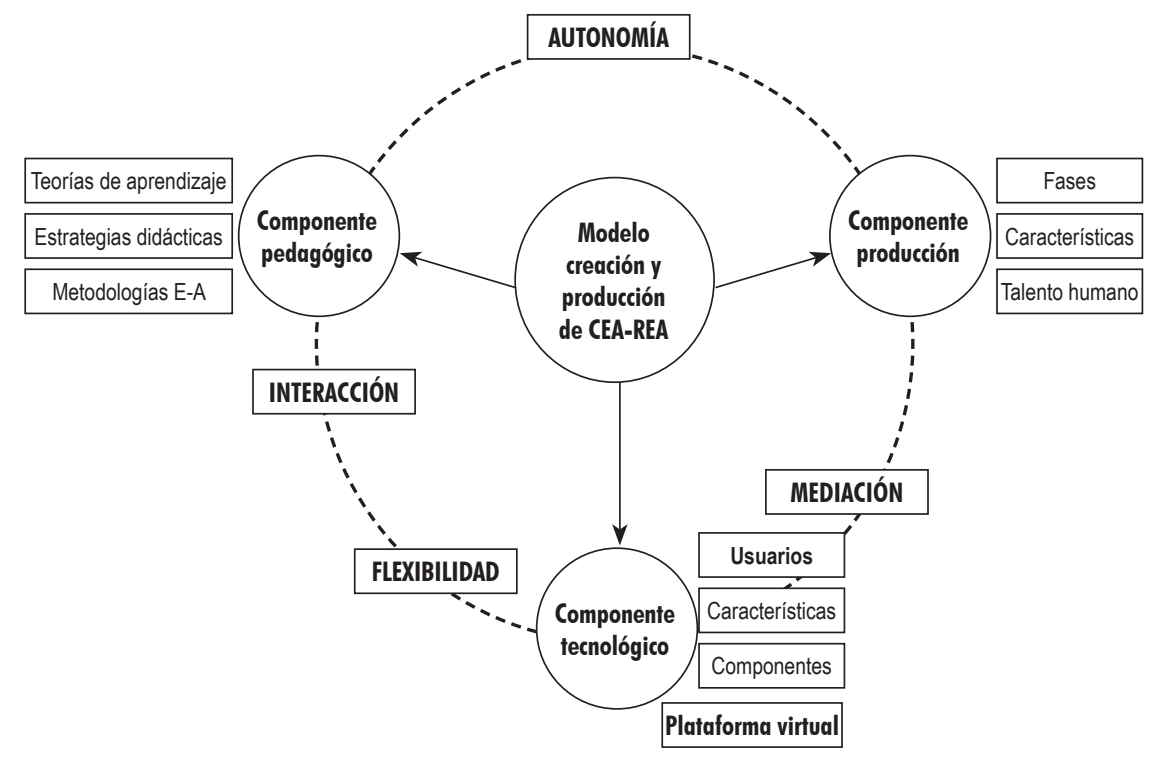

Figura 1. Modelo para la creación y producción de CEA-REA en una plataforma virtual Fuente: elaboración propia.

La interacción se entiende como el proceso de comunicación recíproca entre los participantes de un espacio virtual que puede influir en el comportamiento, el pensar o el actuar de los demás (Vásquez, Ricaurte y Arango, 2009) que incide en la reacción del otro que se evidencia a través de un diálogo o conversación finita, lo cual se demuestra cuando el estudiante tiene una relación recíproca entre los diferentes actores del proceso educativo. Por su parte, la mediación da cuenta del conjunto de acciones o intervenciones, así como de recursos y materiales didácticos como conjunto articulado de componentes que intervienen en el acto educativo y facilitan el proceso de enseñanza y aprendizaje, pues precisa la interacción necesaria entre profesores y estudiantes para ser llevada a cabo.

Desde el principio de la autonomía, se asume que los estudiantes aprenden para la vida y ello les implica acompañamiento por parte del profesor. Además, implica responsabilidad para analizar posibilidades, hacer elecciones y efectuar cambios en la vida que involucran acciones independientes y colaborativas. Desde este punto de vista, la gestión del aprendizaje se encuentra en manos del aprendiz. El docente no hace más que disponerle una serie de herramientas para lograr que dicha gestión sea la más adecuada. Por su parte, la flexibilidad se refiere, según Díaz (2002), a la práctica en la que el aprendiz tiene la posibilidad de escoger o seleccionar la forma, el lugar (espacio) y el momento (tiempo) de su aprendizaje de acuerdo con sus intereses, necesidades y posibilidades. 
Este modelo contempla, además de sus principios, un componente pedagógico que parte del anudamiento de las estrategias y las metodologías de aprendizaje anteriormente enunciadas, con algunas teorías del aprendizaje, como se ilustra en la figura 2:

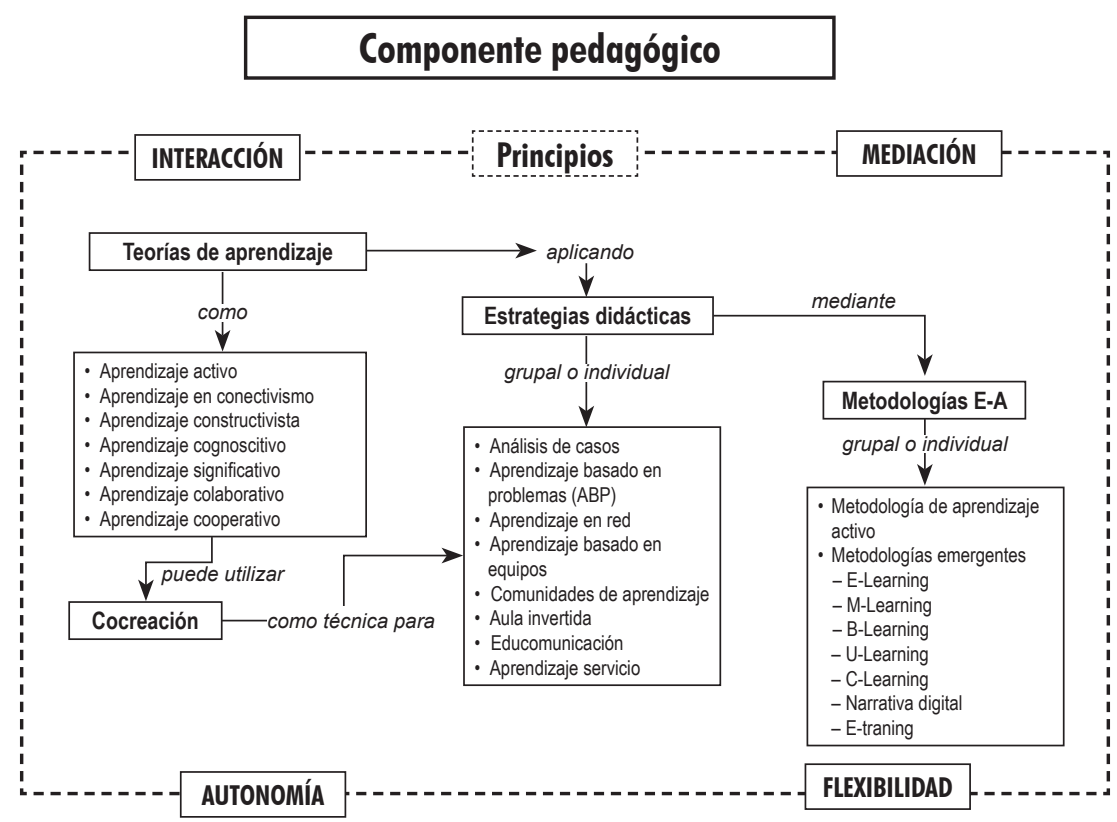

Figura 2. Componente pedagógico

Fuente: elaboración propia.

En este contexto, además de las estrategias pedagógicas implementadas en entornos virtuales de aprendizaje, es importante caracterizar las metodologías de enseñanza y aprendizaje que pueden ponerse en práctica en dichos espacios, pues a través de ellas cobra sentido la incorporación de recursos educativos abiertos al proceso educativo.

De la amplia gama de metodologías de enseñanza-aprendizaje que podrían referirse, este apartado se orientará hacia las metodologías emergentes, es decir, las que se gestan mediante un proceso de integración entre la pedagogía como elemento central y determinante para el desarrollo del aprendizaje en una interacción intencionada del profesor, los contendidos y el estudiante. También se tendrá en cuenta la tecnología o infraestructura de mediación que provee recursos para facilitar la interconexión y gestión de relaciones orientadas a la construcción individual o colectiva del conocimiento. 
De este contexto de relación emergen dinámicas o formas didácticas que estructuran y formalizan nuevas metodologías que facilitan la adaptación y evolución del quehacer educativo en los diversos escenarios donde se gesta el conocimiento. Ante ello, Chatti et al. (2010) sugieren que esta evolución se logra si se parte de las metodologías emergentes hacia unas sustentadas en aprendizaje activo, más personalizadas, sociales, abiertas y dinámicas. Por ello, antes de avanzar con estas metodologías es conveniente adentrase un poco en los planteamientos pedagógicos y metodológicos del aprendizaje activo.

-Aprendizaje activo: las metodologías del aprendizaje activo integran técnicas y estrategias que transforman el proceso de enseñanza-aprendizaje a partir de actividades que propician la participación atenta y activa del estudiante al modificar la forma de entender el aprendizaje y generar cambios profundos en el actuar del profesor. Según afirman Labrador y Andreu (2008), en los recursos de aprendizaje, el profesor y la colaboración que requiere la aplicación de este tipo de metodologías facilitan el desarrollo de comunidades de aprendizaje e interacciones mediadas por entornos virtuales.

De otro lado, promover la construcción colaborativa, la integración de conocimientos previos y las experiencias significativas permite alcanzar aprendizajes más profundos y permanentes. Para lograrlo, según Rué (2007), es necesario que el profesor acoja esta concepción del aprendizaje y que se planifique la enseñanza desde esta transformación. Por ello, el aprendizaje centrado en el estudiante requiere mayor compromiso de su parte porque favorece la autonomía y el desarrollo de competencias para aprender a aprender y aprender con otros en un rol protagónico y autónomo.

En las metodologías emergentes pueden ubicarse, como estrategias que promueven el aprendizaje activo, el aprendizaje basado en problemas (ABP). Este aprendizaje se fundamenta en el aprendizaje, la investigación y la reflexión en busca de respuestas a problemas reales o ficticios. La simulación o aprendizaje en escenarios simulados se refiere a una situación o realidad donde el profesor parte de la investigación, experimentación y reflexión por equipos para el desarrollo autónomo de competencias en un área en particular (Díaz, Leal, y García, 2014). En el mismo sentido, aporta el aprendizaje cooperativo, según Johnson, Johnson y Smith (1991), como una estructura de trabajo en equipo basada en la interdependencia, responsabilidad y desarrollo de habilidades sociales que, al potenciar los conocimientos y habilidades individuales, se orienta hacia el logro de objetivos comunes. Kagan (1985) asocia este aprendizaje con el aprendizaje colaborativo al subscribirlo dentro del aprendizaje constructivista basado en la planificación, diseño 
y construcción de contenidos por parte de los estudiantes hacia la construcción de su propio conocimiento. Sobre estos aspectos planteados del aprendizaje activo y las metodologías que lo propician, se puede dar entrada y sustento didáctico a los diferentes componentes de las metodologías emergentes.

Metodologías emergentes: como se ha planteado ya, se tipifican como emergentes por considerarse el naciente resultado de la evolución de algunas metodologías tradicionales, más concretamente las que se sustentan en las didácticas del aprendizaje activo. Según Chatti et al. (2010) se distinguen por el alto nivel de personalización, dinamismo, apertura e interacción social, y se movilizan a partir del impacto de las tecnologías aplicadas en la educación. La evolución de estas tecnologías se ha dado alrededor de aspectos como la telefonía móvil, soportada en dispositivos portables, su conexión permanente y la disponibilidad de múltiples aplicaciones orientadas a la mediación del aprendizaje.

Se tiene, además, la analítica de aprendizaje, desde la que se agrupan herramientas y técnicas para la obtención, análisis y despliegue de datos referidos a la participación y desarrollo académico del estudiante; la gamificación, representada en tecnologías que incrementan el compromiso del estudiante con mecanismos de juegos en contextos académicos. También hay otros elementos aportantes como tecnologías emergentes que se consideran híbridos, donde se agrupan varias tecnologías que integran e interconectan el mundo físico y el digital, tales como la realidad aumentada, internet de las cosas y dispositivos ponibles.

Lo anterior se complementa con la interacción natural con dispositivos para el caso, como los sistemas de interacción mediante expresiones faciales, gestos y reconocimiento de voz.

Por otra parte, la conjugación de metodologías y estrategias de enseñanzaaprendizaje con los diferentes aportes y potencialidades tecnológicas da lugar a las ya referidas metodologías emergentes, de las que se abordan aquellas que involucran la obtención y construcción de saberes desde una distancia mediada por las tecnologías de información y comunicación. Las principales metodologías emergentes son:

\section{a. Metodología e-learning}

El e-learning es un proceso de enseñanza-aprendizaje llevado a cabo en la interconexión de los estudiantes por medio de recursos tecnológicos. En este sentido, siguiendo los preceptos de la educación a distancia y virtual, Prieto (2013, pp. 25, 37) plantea que en esta modalidad es indispensable tener la habilidad 
del aprendizaje autónomo, el desarrollo del autoconcepto, la autorregulación, la autogestión y la metacognición. Además, sugiere competencias básicas transversales en el área comunicativa, tecnológica y digital, y complementarias en la construcción del ser, la investigación y el emprendimiento. Es una metodología que se fundamenta en el protagonismo, la autonomía y la acción activa del estudiante, el profesor y la interacción grupal que orienta su propósito a llevar el proceso formativo a otros escenarios de aprendizaje fuera del aula tradicional.

Como aspectos de su evolución, esta metodología desvirtúa la dependencia de los recursos físicos para el aprendizaje en la demanda de nuevos elementos, como un computador para la conexión a la web con el ancho de banda y la velocidad necesaria para que el proceso de aprendizaje tenga lugar en escenarios de agilidad, estabilidad y eficiencia.

En su evolución, de los procesos y recursos del e-learning se deriva el m-learning como una tendencia a usar más los dispositivos móviles que los computadores para el acceso a las aplicaciones con propósitos formativos, lo que supone un cambio determinante hacia la ubicuidad del proceso de enseñanza-aprendizaje. A su vez, este proceso se ve limitado por las posibilidades que ofrecen estos dispositivos en cuanto al tamaño de las pantallas, la baja memoria de almacenamiento y la ejecución y restricciones de operación táctil.

\section{b. Metodología m-learning}

El m-learning o aprendizaje con dispositivos móviles se enfoca, en gran parte, en la mediación pedagógica desde los teléfonos inteligentes o tabletas, con la movilidad como principal característica de estas metodologías por la facilidad de conectividad inalámbrica y sus posibilidades telemáticas (Martín et al., 2008).

Para Park, Kim y Lee (2011, pp. 264-271), aunque al respecto son poco los diseños que cuentan con un marco teórico específico, pueden categorizarse desde el soporte del m-learning los enfoques centrados en los niveles cuantitativos y cualitativos de transacción de datos y el análisis desde usuarios agrupados en redes sociales, en oposición a la tendiente individualización y especialización del aprendizaje que se hace posible obtener desde los dispositivos móviles. La gestión del aprendizaje en esta metodología constituye un escenario ideal en el enriquecimiento del ejercicio educativo por estimular la participación en novedosos ambientes de aprendizaje y ofrecer alternativas diferentes al estudiante en la adaptación a la cotidiana realidad tecnológica. Así entonces, la combinación de estos escenarios de aprendizaje en e-learning y la flexibilidad 
que brinda el m-learning, dan lugar a una metodología más efectiva de integración de estrategias no presenciales, con la interacción directa con el profesor (Cloete, 2012).

\section{c. Metodología b-learning}

Es una metodología que, sobre los fundamentos del e-learning o aprendizaje en línea, plantea estrategias que combinan lo presencial y lo virtual en el proceso de enseñanza y aprendizaje. Para Martín et al. (2008), blended learning es un término usado para describir el tipo de e-learning combinado con métodos de enseñanza tradicional en el aula y con el estudio independiente.

El b-learning enfoca sus procesos educativos hacia la mejora de los entornos de enseñanza-aprendizaje al integrar las fortalezas del tradicional aprendizaje sincrónico en tiempo y espacio con actividades en línea asincrónica para favorecer el compromiso del estudiante con el aprendizaje y la interacción (Pahinis et al., 2008).

Para Kavadella et al. (2012, pp. 88-95), la incorporación del blended en el aula sugiere beneficios de interacción, comunicación y comprensión para los estudiantes y profesores. Esta metodología profundiza en los contenidos en desarrollo, mayor colaboración en los proyectos, flexibilidad de tiempo, reducción de espacios y optimización y acceso a los recursos de apoyo. El b-learning supone integrar medios pedagógicos y tecnológicos para fortalecer el acceso a la información, reutilizar y reconvertir recursos físicos en digitales y redistribuir los contenidos hacia la obtención de entornos físicos y virtuales que se fundamentan en los enfoques pedagógicos, herramientas y recursos de interacción.

\section{d. Metodología u-learning}

De acuerdo con Gros y Kinshuk Maina (2016), el aprendizaje ubicuo conjuga las bondades de las metodologías anteriores, bien sea desde el aprendizaje en línea o desde los procesos presenciales en el aula tradicional. Esta metodología puede desarrollar su propósito sin limitantes de tiempo, espacio, dispositivo o contexto. Esto implica, entonces, un conjunto de actividades formativas mediadas por la tecnología móvil, aunque en términos más amplios podría incorporar cualquier medio tecnológico que permita el intercambio de información que pueda representar aprendizaje, lo que incluye medios cotidianos como la televisión, los computadores, los móviles y las tabletas.

El u-learning propone crear escenarios de aprendizaje para la inmersión del estudiante en un ambiente de aprendizaje ubicuo o entorno donde se tenga 
acceso al conocimiento y pueda compartirse con los pares y grupos de interés. Entre sus características más significativas denotamos los contenidos asequibles y disponibles en todo momento y desde cualquier lugar; la permanencia, con actividades de formación en las que se participa, se almacenan y dispone para ser retomadas en procesos futuros de aprendizaje; la colaboración, con posibilidades de comunicación, relación y aprendizaje entre los compañeros, formadores y expertos, bien sea sincrónica o asincrónicamente; la continuidad, esto es, una actividad de aprendizaje a lo largo de la vida como hábito para obtener el conocimiento en el tiempo y la naturalidad que se va generando desde la interacción diaria con lo tecnológico como un proceso transparente e invisible para el estudiante.

e. Metodología c-learning

Es una estrategia para obtener y distribuir contenidos para desarrollar competencias mediante el uso de mecanismos y herramientas ofrecidas por las tecnologías actuales de interconexión, lo que podría llevar a un aprendizaje efectivo fundamentado en la comunidad, la comunicación, la colaboración y la conexión. Se refiere, por tanto, a cualquier aprendizaje logrado por medios sociales o aulas virtuales en un trabajo de pares en comunidad, en escena- rios abiertos al trabajo conjunto y la comunicación, aunque podría incluir también espacios cerrados a cursos privados, participantes limitados o comunidades concretas de interacción. El c-learning es una plataforma abierta de relación horizontal que almacena recursos en la nube y es accesible desde cualquier lugar y dispositivo mediante herramientas gestoras de correo, almacenamiento, comunidad social y demás.

Para Mikroyannidis (2012), el aprendizaje en la nube o c-learning se fundamenta en el aprendizaje comunitario, colaborativo, de exploración y ubicuidad, entre otros, donde el núcleo principal conlleva a la gestión de procesos sociales de construcción de saberes. Estos entornos no son, por lo general, de propiedad de alguien en particular, por lo que profesores y estudiantes son productores y consumidores de recursos y contenidos para la enseñanza y el aprendizaje.

f. Metodología e-training

Esta metodología como la formación empresarial vía e-learning para mejorar la calidad del aprendizaje ante la facilidad de acceso a los recursos y los servicios, los intercambios y la colaboración a distancia en entornos corporativos. Según Bueno (2007), esta metodología se conoce como el e-learning empresarial, en tanto es una solución lógica para alcanzar los objetivos formativos y educativos 
de cada compañía, por lo que una verdadera estrategia de e-training debe centrarse en aquellos aspectos tecnológicos y pedagógicos que enmarquen los métodos de aprendizaje, el modelo pedagógico que la fundamenta y el estudio de aspectos organizacionales como cultura y talento humano.

Dentro del e-training surge, como un método asociado, el e-mentoring, referido a la mentoría electrónica, es decir, telementoring, cybermentoring y online mentoring (Kasprisin et al., 2003). Asimismo, tomando lo planteado por Single y Muller (2001), el e-mentoring da cuenta de la relación entre un individuo senior (mentor) y un individuo menos experimentado o hábil (estudiante). En esta metodología, se usan principalmente comunicaciones electrónicas orientadas a desarrollar y fortalecer sus habilidades, conocimientos, compromiso y comprensión cultural.

\section{Discusión y conclusiones}

En el apartado anterior se mostró la correlación entre estrategias, metodologías y teorías de aprendizaje como fundamento pedagógico del modelo de producción de REA investigado. Dicha correlación da lugar a lo que Medina (2003, citado por Mayorga y Madrid, 2010, p. 93) define como doble vertiente. Esto quiere decir que el modelo propuesto es a la vez anticipador y previo a la práctica educativa, lo que permite dotar de sentido y pertinencia a cada acción e iniciativa que tiene lugar en el ámbito académico, no solo de cara al maestro en tanto es generador de dichas iniciativas, sino también por la responsabilidad que transmite al estudiante en su propio proceso.

Vale la pena mencionar que este modelo puede clasificarse, de acuerdo con Mayorga y Madrid (2010), como un modelo didáctico-tecnológico en la medida que "combina la preocupación de transmitir el conocimiento acumulado con el uso de metodologías activas. Existe preocupación por la teoría y la práctica, de manera conjunta" (p. 95). En este sentido, revela la preocupación de los grupos de investigación y la empresa participante que confluyeron en su propuesta por acercar la realidad educativa a procesos educativos más democráticos, de acceso abierto, centrados en las necesidades que la modernidad va signando para la educación de cara a los procesos de calidad que no solo se preguntan por la incidencia de la sociedad de la información en la cotidianidad, sino también en los ámbitos educativos.

Desde la integración entre los procesos pedagógicos y las mediaciones tecnológicas, se gestan didácticas y metodologías que dan cuenta de la evolución en el proceso de enseñanza-aprendizaje mediante escenarios diversos y abiertos para la gestión de los saberes. Por ello, en acuerdo con Chatti et al. (2010), será posi- 
ble lograr dicha evolución si se fundamenta esta corelación en las metodologías emergentes sobre los planteamientos del aprendizaje activo, en tanto sugiere un proceso personalizado, social, abierto y dinámico que se nutre con la autonomía y autorregulación de quien aprende bajo un acompañamiento de orientación por parte del profesor. Como complemento, acogemos las ideas de Labrador y Andreu (2008) en cuanto a que los recursos, el profesor y la colaboración que demandan estas metodologías estimulan y promueven, de alguna manera, la constitución de comunidades y procesos de interacción para el aprendizaje en los entornos virtuales.

Podemos tipificar, entonces, las metodologías emergentes como escenarios de aprendizaje orientados a un proceso personal, dinámico y abierto a la interacción y colaboración para la construcción de un conjunto de conocimientos en una mediación desde dispositivos y formas diversas de interconexión tecnológica. En este sentido, nos sumamos a lo expuesto por Prieto $(2013$, pp. 25,37$)$ cuando considera indispensable que, en la metodología e-learning, se cuente con la habilidad del aprendizaje autónomo, el desarrollo del autoconcepto, la autorregulación, la autogestión y la metacognición. Pero no podemos quitarle fuerza y fundamento al papel que juegan las interacciones y las posibilidades abiertas de construcción conjunta en cuanto al potencial de construir, confrontar y llegar a consensos conceptuales con pares y expertos en la disciplina.

La discusión frente estas metodologías podría partir del hecho de que, independiente de las metodologías emergentes que se opte por implementar en el proceso de enseñanza aprendizaje, será un factor determinante del éxito las capacidades del estudiante para acogerse a las nuevas dinámicas que ello implica. En la tradicional forma didáctica no se ha avanzado lo suficiente para encontrar, ante esta exigencia, estudiantes autónomos, participativos, críticos y dispuestos a una construcción abierta y conjunta con otros. Además de lo que implica la apropiación e incorporación tecnológica, debemos considerar, y con mayor fundamento, la falta de competencias comunicacionales y cognitivas en la población estudiantil.

Es determinante para el aprendizaje, bajo la concepción de las metodologías emergentes, dejarse permear por los conceptos de personalización, dinamismo, autonomía y autorregulación que plantea el aprendizaje activo para poder pensar en una construcción conjunta que resulte de la interconexión, interacción y trabajo colaborativo. Las metodologías emergentes, en su fundamento de interacción y conectividad, demandan en los actores del proceso de enseñanza-aprendizaje competencias comunicativas, tecnológicas, cognitivas y de trabajo colaborativo. 
Aunque las metodologías emergentes sustentan sus acciones en la tecnología e interacción, tener estas competencias no garantiza la efectividad de un proceso de aprendizaje porque el rol de quien aprende y enseña debe reinventarse en su concepción y procesos cognitivos y pedagógicos.

Ahora que los conceptos de espacio, tiempo y alcance se ven transformados en la conjugación tecnopedagógica, es necesario el desarrollo de otras formas de aprendizaje para otros propósitos y con mediaciones diferentes que implican transcender las limitaciones de la educación, el aprendizaje y la enseñanza misma.

Al momento de plantear escenarios de aprendizaje colaborativo, es necesario abordar inicialmente las teorías de aprendizaje individual con el fin de establecer factores y ambientes que propicien el conocimiento a ese nivel, así como nutrir y hacer efectivo el trabajo colaborativo de construcción de saberes.

Para la generación del modelo, resultado de esta investigación, se tuvieron en cuenta tres aspectos básicos para la elaboración de un RED, estos son: i) lo educativo, donde se estable la intención pedagógica del RED; ii) Lo tecnológico, que permite su transformación en un recurso digital, iii) lo abierto, que permite la disponibilidad de los recursos en un lugar de acceso público (Arango y Manrique, 2020).

Para concluir, vale la pena señalar que es necesario cambiar la forma de enseñar y de adquirir conocimiento con profesores que exploren cada día nuevas formas de enseñar a través de las diferentes herramientas de las tecnologías de la información y la comunicación (TIC), que en la actualidad están tan ligadas con la educación. Además, con el trabajo conjunto que se realice en el aula de clase, se permitirá a los estudiantes generar nuevos conocimientos en las que se exploren estrategias que les ayuden a solucionar situaciones de la vida real gracias a la guía que le brinda el profesor en todos los procesos de educativos.

\section{Resumen de los hallazgos y aportes al área de conocimiento}

En la búsqueda de metodologías y estrategias de enseñanza-aprendizaje orientadas al trabajo autónomo, el aprendizaje significativo, la colaboración y la solución de problemas desde el sustento de las teorías pedagógicas y sus planteamientos frente al rol del estudiante y el profesor, la disposición de los contenidos, las formas de interacción, los recursos utilizados, los propósitos e intenciones didácticas, las tipologías de actividad y sus posibilidades de aplicación en una plataforma virtual; se encuentra que los planteamientos del aprendizaje activo dan lugar a metodologías que integran técnicas y estrategias para la transformación del proceso de enseñanza-aprendizaje mediante actividades que propician la participación atenta 
y activa del estudiante al modificar la forma de entender el aprendizaje y generar cambios profundos en el rol del profesor.

En este sentido, se encuentran estrategias como el aprendizaje colaborativo basado en equipos, el aula invertida, el análisis de casos, el aprendizaje basado en proyectos, la educomunicación, comunidades de aprendizaje y el aprendizaje-servicio como estrategias que, por sus planteamientos y roles del profesor y estudiante frente al aprendizaje autónomo y la construcción conjunta de saberes desde los conocimientos previos, la reflexión, investigación y hacia la solución de problemas, constituyen aquellas estrategias propias para el desarrollo de conocimiento en un ambiente de aprendizaje basado en plataformas educativas virtuales.

De igual manera, se encuentran metodologías emergentes que plantean, desde los principios de las metodologías tradicionales, nuevas formas de comunicación, roles para el profesor y estudiante e interacciones orientadas al aprendizaje. Al estar mediadas por las tecnologías de información y comunicación, integran posibilidades más flexibles, abiertas e inclusivas en los procesos de enseñanza y aprendizaje en línea con variaciones en su intencionalidad y combinaciones entre los momentos y escenarios de aprendizaje en línea o presencial. Esta integración e interconexión del mundo físico y el digital en interacciones mediadas por realidad aumentada, internet de las cosas y dispositivos ponibles permiten el acceso al conocimiento en cualquier tiempo y lugar.

Estas estrategias y metodologías encontradas como objeto de la investigación para el propósito planteado, aportan de manera significativa en los procesos de aprendizaje, en tanto sus propuestas constituyen hoy en día aspectos determinantes para potenciar las bondades de la tecnología en la educación. Desde la conectividad integradora, las posibilidades de acceso, la diversidad de presentación de la información y las necesidades de cobertura e inclusión en la educación, estas van iniciando nuevas formas de desarrollo del conocimiento en las formas de acceder, apropiar y aplicar los saberes adquiridos.

Asimismo, son estrategias y metodologías que aportan a nuevos procesos de oferta académica, basadas en la autorregulación, la responsabilidad y la necesidad puntual de aprehensión de un saber específico y puntual por parte quien aprende. En este contexto, la información estará publicada en todas partes y al alcance de todos. Las necesidades de aprendizaje serán, entonces, cada vez más variadas y específicas. Los profesores o tutores se verán obligados a orientar, dirigir, canalizar y promover el logro de objetivos puntuales con recursos digitales cada vez más amplios en sus expresiones y con mayores posibilidades cognitivas para quienes los aborden, donde las comunidades de aprendizaje serán determinantes como escenarios de 
interacción, construcción conjunta, validación y confrontación de saberes. Además, constituirán, de alguna manera, una ampliación del aula o escenarios tradicionales que trascenderán el aprendizaje. De esta manera, se posibilitará la práctica misma o la conexión con escenarios reales o virtuales de entrenamiento gracias a la integración entre empresarios, científicos, académicos y estudiantes que podrán satisfacer, desde estas comunidades de desarrollo y aplicación de conocimiento, sus necesidades investigativas, productivas y de aprendizaje.

\section{Referencias}

Arango, S y Manrique, B. (2020, 27 de junio). Virtual Platform for the Institución Universitaria Digital de Antioquia-Colombia [ponencial 15th Iberian Conference on Information Systems and Technologies (CISTI), Sevilla, España. https://ieeexplore.ieee.org/document/9141066

Boaden, R. (2006). Leadership development: does it make a difference? Leadership \& Organization Developement Journal, 27(1), 5-27. https://www.researchgate.net/ publication/235250453_Leadership_development_Does_it_make_a_difference

Bonwell, C. C. y Eison, J. A. (1991). Active Learning: Creating Excitement in the Classroom. (ASHE-ERIC Higher Education Report No. 1). The George Washington University.

Bottorff, J. y Morse J. (2003). Asuntos críticos en los métodos de investigación cualitativa. Universidad de Antioquia.

Bowerman, J. (2003). Leadership development through action learning: an executive monograph. International Journal of Health Care Quality Assurance, 16(4), 6-14. http://dx.doi. org/10.1108/13660750310500049

Bueno, J. A. (2007). Propuesta metodológica para la implementación de una estrategia e-training. Gerencia de Tecnologías de Información, 6(16). https://core.ac.uk/download/pdf/230227198.pdf

Chatti, M. A., Agustiawan, M. R., Jarke, M. y Specht, M. (2010). Toward a personal learning environment framework. International Journal of Virtual and Personal Learning Environments, 1(4), 66-85. https://pdfs.semanticscholar.org/522d/6200a97357a345a39f95da0910e6d186 ed2d.pdf

Cloete, L. (2012). Combining E-learning and M-learning: new applications of blended educational resources. Onlineinformationreview, 36(2),321-322.https://doi.org/10.1108/14684521211240153

Conger, J. y Toegel, G. (2002). Action learning and multi-rater feedback as leadership development interventions: Popular but poorly deployed. Journal of Change Management, 3(4), 332348. https://www.researchgate.net/publication/233679749

Cornide-Reyes, Héctor C., y Villarroel, Rodolfo H. (2019). Método para Promover el Aprendizaje Colaborativo en Ingeniería de Software. Formación universitaria, 12(4), 3-12. https://dx.doi. org/10.4067/S0718-50062019000400003

Díaz, J. L., Leal, C. y García, J. A. (2014). Metodología de autoaprendizaje en entornos simulados (MAES). Evidentia, 11, pp. 1-6. https://www.researchgate.net/publication/303231958_Metodologia_de_autoaprendizaje_en_entornos_simulados_MAESC 
Díaz, M. (2002). Flexibilidad y educación superior en Colombia. Icfes (1 ${ }^{\mathrm{a}}$ ed.).

Downes, S. (2007, 3 de febrero). What Connectivism is. https://halfanhour.blogspot.com/2007/02/ what-connectivism-is.html

Freeman, S.,Sarah, L., McDonough, M., Michelle, K., Okoroafor, N., Jordt. H., y Wenderoth, M. (2014). Active learning increases student performance in science. Engineering. And mathematics. Psychological and cognitive sciences. Ed.Bruce Alberts, Univ. Califormina.

Gadamer, H. G. (1992). Verdad y método II. Ediciones Sígueme.

Gairín, J. (2006). Las comunidades virtuales de aprendizaje. Educar, (37). 41-64.

Galeano, M. (2004). Diseño de proyectos en la investigación cualitativa. Fondo Editorial Universidad Eafit.

Gil-Galván, R. (2018). El uso del aprendizaje basado en problemas en la enseñanza universitaria. Análisis de las competencias adquiridas y su impacto. Revista mexicana de investigación educativa, 23(76), 73-93. https://www.redalyc.org/articulo.oa?id=14054854004

González, E. (2006). Sobre la hermenéutica o acerca de las múltiples lecturas de lo real. Sello Editorial Universidad de Medellín.

Gros, B. y Kinshuk Maina, M. (2016). The Future of Ubiquitous Learning: Learning Desings for Emerging Pedagogies. Lecture Notes in Educational Technology. The Future of ubiquitous Computing. Springer International Publishing. http://doi.org/10.1007/978-3-662-47724-3

Johnson, D. W., Johnson, R. T. y Smith, K. A. (1991). Cooperative learning: Increasing college faculty instructional productivity. The George Washington University.

Kagan, S. (1985). Cooperative learning resources for teachers. University of California.

Kasprisin, C. A., Single, P. B., Single, R. M. y Muller, C. B. (2003). Building a Better Bridge: testing e-training to improve e-mentoring programmes in higher education. Mentoring $\&$ Tutoring, 1 (11). https://doi.org/10.1080/1361126032000054817

Kavadella, A., Tsiklakis, K., Vougiouklakis, G. y Lionarakos, A. (2012). Evaluation of a blended learning course for teaching oral radiology to undergraduate dental students. Eur J Dent Educ, (16), 88-95. https://pubmed.ncbi.nlm.nih.gov/22251359/

Labrador, M. y Andreu, M. (2008). Metodologías activas. Grupo de innovación en Metodologías Activas. Ediciones Universidad Politécnica de Valencia. http://www.upv.es/diaal/publicaciones/Andreu-Labrador12008_Libro\%20Metodologias_Activas.pdf

Martín, S., Gil, R., Díaz, G., Sancristobal, E., Castro, M. y Peire, J., (2008,15 de octubre). From elearning to m-learning through b-learning and s-learning. 50th International Symposium Elmar, Zadar, 341-344. https://www.researchgate.net/publication/224370701_From_e-learning_ to_m-learning_through_b-learning_and_s-learning

Martínez, I., Martínez, B., Alonso-Sáez, I. y Gezuraga, M. (2013). El Aprendizaje-Servicio en la innovación universitaria. Una experiencia realizada en la formación de educadoras y educadores sociales. Revista Internacional de Educación para la Justicia Social, 2, 195-216. https:// revistas.uam.es/riejs/article/view/379 
Mayorga, M. y Madrid, D. (2010). Modelos didácticos y Estrategias de enseñanza en el Espacio Europeo de Educación Superior. Tendencias Pedagógicas 15(1). https://dialnet.unirioja.es/ descarga/articulo/3221568.pdf

Mcgrath, D. (2002). Teaching on the front lines: using the internet and problem-based learning to enhance classroom teaching. Holist Nurs Pract, 16(2), 5-13. https://www.researchgate. net/publication/11515061

Michaelsen, K., Davidson, N. y Major, C. (2014). Team Based Learning Practices and Principles in Comparison with Cooperative Learning and Problem Based Learning. Journal on Excellence in College Teaching 25(3 y 4), 57-84. https://www.lhthompson.com/uploads/4/2/1/1/42117203/ team_based_learning___group_work.pdf

Mikroyannidis, A. (2012). A Semantic Framework for Cloud Learning Environments. In Chao, L. (Eds.), Cloud Computing for Teaching and Learning: Strategies for Design and Implementation (pp. 17-31). IGI Global. http://doi:10.4018/978-1-4666-0957-0.ch002

Pahinis, K., Stokes, C. W., Walsh, T. F., Tsitrou, E. y Cannavina, G. A. (2008). Blended learning course taught to different groups of learners in a dental school: Follow-up evaluation. J Dent Educ., 72(9), 57-1048. 10.1002/j.0022-0337.2008.72.9.tb04579.x

Paredes, D. (2019). Dimensiones pedagógicas que configuran las prácticas de aprendizaje-servicio. Páginas de Educación, 12(2), 23-42. https://dx.doi.org/10.22235/pe.v12i2.1834

Park, S., Kim, K. y Lee, B. (2011). Developing english learning contents for mobile smart devices. future Information Technology, 185, 264-271. https://link.springer.com/chapter/10.1007\% 2F978-3-642-22309-9 32

Prieto, J. (2013). Una aproximación metodológica al uso de redes sociales en ambientes virtuales de aprendizaje, para el fortalecimiento de las competencias transversales de la Universidad EAN. Revista Virtu@Imente, 1(1),25-31. https://journal.universidadean.edu.co/index. php/vir/article/view/1397

Reyes-Torres, G., Saavedra, J. y Aguayo-Vergara, M. (2020). Aprendizaje basado en equipos en un curso de Ingeniería en Educación Superior. Revista Educación, 44(1), pp. 257-276. https:// dx.doi.org/10.15517/revedu.v44i1.38316

Rué, J. (2007). Enseñar en la universidad. El EEES como reto para la educación superior. Narcea.

Schneider, E., Froze, I., Rolon, V., y Mara de Almeida, C. (2013). Sala de Aula Invertida. En EAD: Uma proposta de Blended Learning. Revista Intersaberes, 8(16), 68-81. https://www.uninter. com/intersaberes/index.php/revista/article/view/499

Siemens, G. (2006). Conectivism: learning and knowledge today. http://www.mmiweb.org.uk/egyptianteachers/site/downloads/Siemens_2006.pdf

Siemens, G. (2008). A learning theory for the digital age. https://www.academia.edu/2857237/ Connectivism_a_learning_theory_for_the_digital_age

Single, P. B. y Muller, C. B. (2001). When e-mail and mentoring unite: The implementation of a nationwide electronic mentoring program, in: L. Stromei (ed.) Creating Mentoring and Coaching Programs (pp.107-121). American Society for Training and Development in Action Series. 
Unesco. (Octubre de 2002). La educación superior en el siglo XXI: visión y acción. Presentada en la Conferencia Mundial sobre la Educación Superior en el Siglo XXI, en París. https://unesdoc.unesco. org/ark:/48223/pf0000116345_spa

Vásquez, C., Ricaurte, A. y Arango, S., (2009). Interacciones comunicativas en un entorno virtual de aprendizaje. Revista Virtual Universidad Católica del Norte, 28. 